\title{
Coronary artery disease screening and prognosis in incident dialysis patients
}

\author{
Yuri Tanaka ${ }^{1+}$, Nobuhiko Joki ${ }^{{ }^{*}+}$, Toshihide Hayashi ${ }^{1}$, Masaki Iwasaki ${ }^{1}$, Shun Kubo ${ }^{1}$, Takasuke Asakawa ${ }^{1}$, \\ Ai Matsukane', Mari Horie', Yasunori Takahashi ${ }^{2}$, Hiroki Niikura ${ }^{4}$, Koichi Hirahata ${ }^{3}$, Yoshihiko Imamura ${ }^{2}$ \\ and Hiroki Hase ${ }^{1}$
}

\begin{abstract}
Background: Guidelines have recommended that physicians screen for coronary artery disease (CAD) at the initiation of dialysis. The purpose of this study was to examine the effects of CAD screening at the initiation of dialysis on prognosis after starting dialysis

Methods: This retrospective cohort study involved 224 consecutive incident dialysis patients without advanced cardiac disease. Pharmacological stress myocardial perfusion imaging (MPI) was performed to routinely screen incident dialysis patients within 3 months of starting dialysis therapy. The influence of MPI on prognosis after starting dialysis was explored by the propensity score adjustment method. The study outcome was defined as all-cause mortality and cardiac death.
\end{abstract}

Results: Of the 224 patients, 164 (73.2 \%) underwent MPI screening at initiation of dialysis. During the median follow-up period of 5.5 years, 77 patients died, among whom 17 (22.1 \%) cardiac deaths were observed. The patients who received MPI had a significantly higher survival rate for all-cause mortality and cardiac death compared with the patients without MPI (log-rank test, $p<0.001, p=0.021$, respectively). In multiple Cox regression analysis, MPI screening continued to have a statistically significant negative association with all-cause mortality after adjusting for confounding variables (hazard ratio $0.581, p=0.034$ ) and propensity score (hazard ratio 0.568 , $p=0.025$.

Conclusions: MPI CAD screening at dialysis initiation could improve prognosis after starting dialysis.

Keywords: Myocardial perfusion imaging, Propensity score, Initiation of dialysis, All-cause mortality, Cardiac death

\section{Background}

Although the underlying mechanism of the cardio-renal axis in patients with chronic kidney disease (CKD) has been determined in considerable detail over the past decade [1], cardiovascular disease is still a leading cause of death in maintenance dialysis patients worldwide [2, 3] and also in Japan [4]. The accelerated progression of atherosclerosis during the conservative phase of CKD, before starting dialysis therapy [5], is thought to be one of the causes of the high incidence of cardiovascular events in the dialysis phase $[6,7]$. Therefore, it is well

\footnotetext{
*Correspondence: jokinobuhiko@gmail.com

${ }^{\dagger}$ Equal contributors

'Department of Nephrology, Toho University Ohashi Medical Center, 2-17-6 Ohashi, Meguro-ku, Tokyo 153-8515, Japan

Full list of author information is available at the end of the article
}

known that a high prevalence of atherosclerotic cardiovascular diseases is observed at the initiation of dialysis. For example, approximately $50 \%$ of incident asymptomatic dialysis patients have been observed to suffer from advanced coronary artery disease (CAD) $[8,9]$ and show poor prognosis after starting dialysis $[6,10]$. Based on this background, guidelines created by several organizations [11-13] have recommended that physicians screen for CAD at the initiation of dialysis in order to find it earlier and improve prognosis. However, it has not yet been established whether screening for CAD plays a crucial role in improving the prognosis for dialysis patients.

Pharmacological stress myocardial perfusion imaging (MPI), even in end-stage kidney disease (ESKD) patients, is one of the most frequently performed procedures for identifying patients with myocardial ischemia and CAD, 
and for stratifying the patients at high risk for poor prognosis [14]. Recently, the severity of abnormal MPI results has begun to have a great impact on the prediction of cardiac events in the conservative phase of advanced CKD patients without cardiovascular disease [15]. Therefore, MPI is becoming a useful screening procedure for patients at any stage of kidney disease as a non-invasive procedure that is not harmful to kidney function.

Our hypothesis for the present study was that screening for CAD at the initiation of dialysis by using MPI may lead to improved prognosis after starting dialysis. Therefore, this study examined the effect of MPI screening for CAD on the prognosis of incident dialysis patients.

\section{Methods}

\section{Study design and patients}

This was a retrospective cohort study that involved 314 consecutive patients who started maintenance dialysis for ESKD at the Toho University Ohashi Medical Center between January 2001 and December 2010. In order to precisely evaluate the effect of MPI screening on prognosis, ESKD patients who already had cardiac disease before starting dialysis were excluded. As shown in Fig. 1, 74 patients met the exclusion criteria as follows: (1) history of New York Heart Association (NYHA) level III or IV heart failure, (2) coronary heart disease such as myocardial infarction and/or coronary revascularization, or (3) left ventricular dysfunction defined as ejection fraction (EF) $<50 \%$ evaluated by echocardiography. Additionally, 16 patients who expired within 3 months of starting dialysis were excluded. The remaining 224 patients were enrolled into this study, among whom 164 underwent MPI as screening for CAD. The influence of MPI on prognosis after starting dialysis was explored by crude cohort analysis and by the propensity score adjustment method.

All patients provided informed consent to participate in the study, and the Ethics Committee for Clinical Research at Toho University Ohashi Medical Center approved the study protocol [Permission no. 13-52, 13-61]. This study adhered to the Declaration of Helsinki.

\section{Data collection}

Clinical information from all patients was recorded before the first hemodialysis (HD) session. All patients

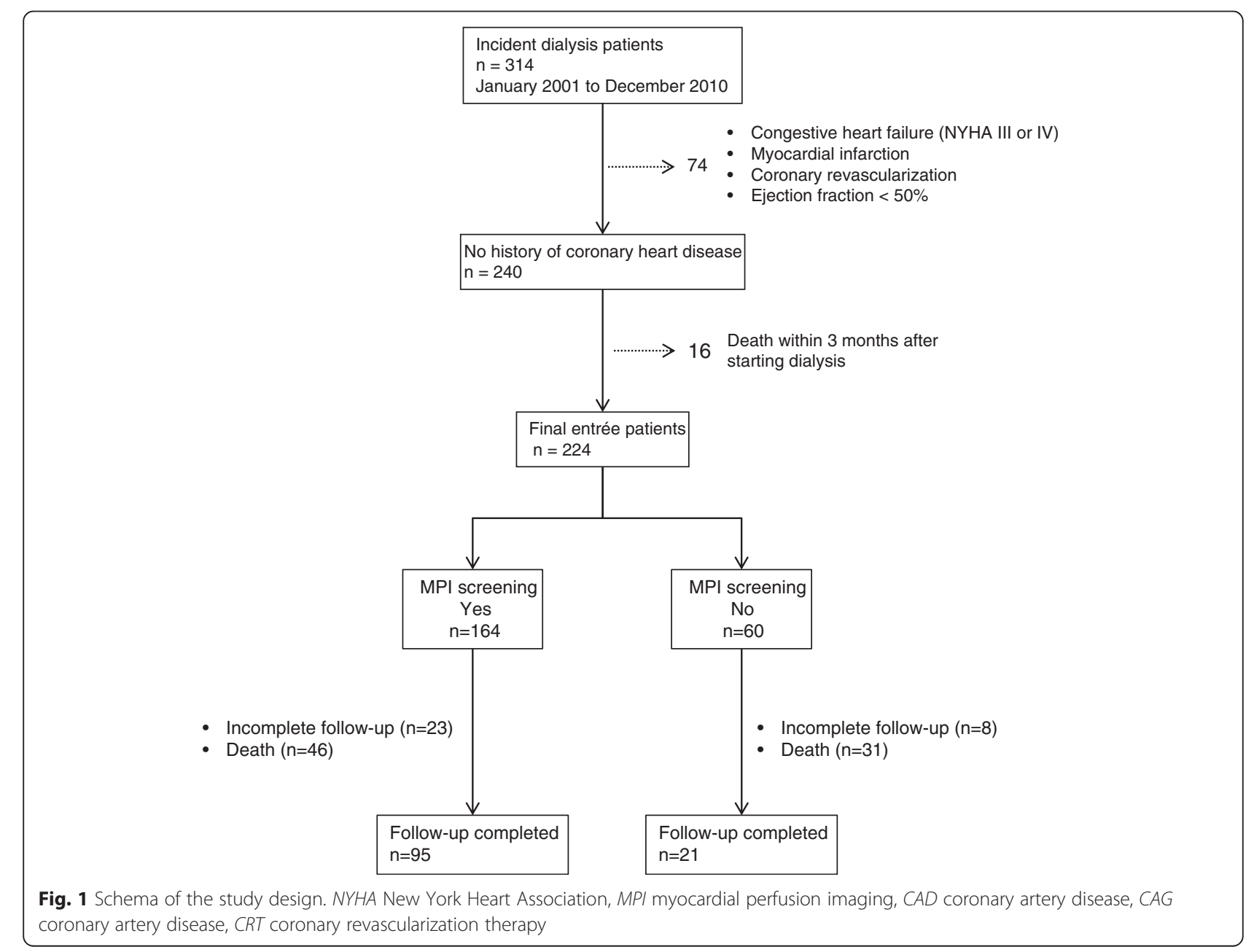


were initially interviewed to determine age, sex, type of primary renal disease, previous hospitalization, and history of hypertension and cardiac disease. We also collected information about the medications the patients were receiving after starting dialysis. Blood pressure was recorded from the supine position, and blood samples were withdrawn immediately before the first HD session. Serum calcium $(\mathrm{Ca})$ concentrations were corrected by albumin levels as follows: corrected $\mathrm{Ca}(\mathrm{cCa})=$ measured $\mathrm{Ca}(\mathrm{mg} / \mathrm{dL})+(4-$ measured albumin level $(\mathrm{g} / \mathrm{dL}))$. The estimated glomerular filtration rate (eGFR) was calculated using the Japanese equation as follows [16]: eGFR $\left(\mathrm{mL} / \mathrm{min} / 1.73 \quad \mathrm{~m}^{2}\right)=194 \times \mathrm{Cr}^{-1.094} \times \mathrm{Age}^{-0.287} \quad$ (for women $\times 0.739$ ). Body mass index $(\mathrm{BMI})$ at optimal weight was calculated as weight $(\mathrm{kg})$ divided by height (m) squared. All participants were assessed by echocardiography at optimum weight after a dialysis session or on an intermittent day of hemodialysis before discharge. Ejection fraction was measured as a marker of left ventricular (LV) dysfunction.

\section{MPI as a screening method for coronary artery disease} From January 2001, ESKD patients at our hospital have been routinely screened for CAD within 3 months of starting HD therapy, regardless of any suspicion for ischemic heart disease (IHD). Pharmacological stress MPI by using thallium-201 was performed for screening in 164 of the 224 ESKD study patients. Clinically significant CAD was defined when myocardial perfusion defects were reversible in patients examined by pharmacological stress thallium-201 MPI.

\section{Follow-up}

The outcome of this study was defined as all-cause mortality and cardiac death. Cardiac death was defined as death from heart failure, myocardial infarction, or sudden death. Information about death was obtained from a questionnaire survey sent to the maintenance hemodialysis facilities or the hospital medical records. The cohort was followed until the end of 2012.

\section{Statistical analysis}

Data are summarized as number, prevalence, arithmetic mean \pm standard deviation, or median (interquartile range), as appropriate. Comparisons of prevalence and values between groups were performed by Student's $t$ test and the $\chi^{2}$ test. In the prospective analysis, the Kaplan-Meier method was used with the log-rank test. Prognostic variables were examined by Cox proportional hazard models, and hazard ratios and $95 \%$ confidence intervals are reported. $p$ values $<0.05$ were considered significant. To adjust for differences in baseline characteristics between the two patient groups (with and without screening MPI), a propensity score analysis was performed using multivariate logistic regression modeling, including all baseline covariates such as age, sex, diabetes, BMI, systolic blood pressure (SBP), LV EF, albumin, hemoglobin $(\mathrm{Hb})$, total cholesterol $(\mathrm{TC})$, triglyceride (TG), high-density lipoprotein cholesterol (HDL$\mathrm{C})$, eGFR, cCa, phosphate (P), intact-parathyroid hormone (iPTH), c-reactive protein (CRP), and usage of three medications: renin angiotensin aldosterone system inhibitor (RAS-I), beta blocker (BB), and statin. The score was then incorporated into a Cox proportional hazards model as a covariate. All statistical analyses were performed using SPSS for Windows version 20 (IBM, Tokyo, Japan).

\section{Results}

\section{Baseline characteristics}

Of the 224 study patients, 164 (73.2 \%) patients underwent MPI screening at the initiation of dialysis (Table 1). Significantly higher incidence of diabetes, higher diastolic pressure, and higher creatinine concentration (lower eGFR) were observed in the MPI screening group compared with the non-MPI group. Although the MPI group had a younger age, more males, higher SBP, lower $\mathrm{Hb}$, higher HDL-C, lower $\mathrm{cCa}$, and higher $\mathrm{P}$ than the non-MPI group, no statistically significant differences were found. Among the 164 patients who underwent the screening, 27 patients (16.5\%) showed abnormal MPI. The usage of three medications (statin, RAS-I, and BB) did not differ significantly between the two groups.

\section{Follow-up}

The median follow-up period was 5.5 years. During the follow-up period, 77 patients died, including 17 cardiac deaths (22.1\%), as shown in Table 2. A total of 31 patients were lost to follow-up, and two patients received renal transplantation and were censored at the time of the last observation, which was the operation day for renal transplantation (Fig. 1). At the end of the followup period on December 31, 2012, a total of 114 patients were alive on maintenance hemodialysis and had completed follow-up.

As shown in Fig. 2a, the Kaplan-Meier curves indicated that the patients with MPI screening had a significantly higher survival rate than the patients who did not undergo MPI screening (log-rank test, $p<0.001$ ). The cumulative survival rates at 5 years after starting dialysis with and without MPI were 74.9 and $49.1 \%$, respectively. Regarding cardiac death in Fig. $2 b$, the cardiac death event-free 5-year survival rate was $94.8 \%$ in patients with the screening and $86.6 \%$ in patients without the screening (log-rank test, $p=0.021$ ). After adjusting by propensity score, the Kaplan-Meier curves still showed higher survival in patients with MPI than in those without MPI (dotted line in Fig. 2a, b). 
Table 1 Baseline characteristics of incident dialysis patients with and without MPI screening

\begin{tabular}{|c|c|c|c|}
\hline & \multicolumn{2}{|c|}{ MPI screening } & \multirow{3}{*}{$p$} \\
\hline & \multirow{2}{*}{$\begin{array}{l}\text { Yes } \\
n=164\end{array}$} & \multirow{2}{*}{$\begin{array}{l}\text { No } \\
n=60\end{array}$} & \\
\hline & & & \\
\hline Age (years) & $64 \pm 12$ & $66 \pm 17$ & 0.350 \\
\hline Male (\%) & 67 & 55 & 0.097 \\
\hline BMI $\left(\mathrm{kg} / \mathrm{m}^{2}\right)$ & $22 \pm 3$ & $22 \pm 3$ & 0.170 \\
\hline Diabetes (\%) & 55 & 37 & 0.016 \\
\hline $\mathrm{SBP}(\mathrm{mmHg})$ & $154 \pm 23$ & $147 \pm 30$ & 0.069 \\
\hline $\mathrm{DBP}(\mathrm{mmHg})$ & $78 \pm 16$ & $72 \pm 11$ & 0.014 \\
\hline Ejection fraction (\%) & $67 \pm 9$ & $66 \pm 9$ & 0.403 \\
\hline Albumin (g/dL) & $3.3 \pm 0.5$ & $3.3 \pm 0.5$ & 0.525 \\
\hline Hemoglobin (g/dL) & $8.1 \pm 1.5$ & $8.4 \pm 1.6$ & 0.260 \\
\hline Total cholesterol (mg/dL) & $175 \pm 53$ & $177 \pm 55$ & 0.828 \\
\hline Triglyceride (mg/dL) & $123 \pm 72$ & $132 \pm 61$ & 0.378 \\
\hline $\mathrm{HDL}-\mathrm{C}(\mathrm{mg} / \mathrm{dL})$ & $50 \pm 17$ & $46 \pm 15$ & 0.172 \\
\hline Creatinin (mg/dL) & $9.8 \pm 3.5$ & $8.6 \pm 3.9$ & 0.037 \\
\hline eGFR (mL/min/1.73 m²) & $5.0 \pm 1.7$ & $5.8 \pm 2.3$ & 0.007 \\
\hline $\mathrm{cCa}(\mathrm{mg} / \mathrm{dL})$ & $8.0 \pm 1.0$ & $8.2 \pm 0.9$ & 0.107 \\
\hline$P(m g / d L)$ & $6.2 \pm 1.7$ & $5.8 \pm 1.6$ & 0.082 \\
\hline iPTH (pg/mL) & $338 \pm 315$ & $302 \pm 170$ & 0.396 \\
\hline CRP (mg/dL) (IQR) & 0.1 & 0.15 & 0.154 \\
\hline Statin (\%) & 18 & 19 & 0.953 \\
\hline RAS inhibitor (\%) & 66 & 53 & 0.071 \\
\hline Beta blocker (\%) & 23 & 17 & 0.321 \\
\hline Coronary artery disease (\%) & $27(16.5)$ & & \\
\hline CRT (\%) & $9(5.5)$ & & \\
\hline
\end{tabular}

$B M I$ body mass index, $C R P$ c-reactive protein, $C R T$ coronary revascularization therapy, SBP systolic blood pressure, DBP diastolic blood pressure, eGFR estimated glomerular filtration rate, HDL-C high-density lipoprotein cholesterol, RAS renin angiotensin system

Figure 3 depicts the survival curves in three patient groups: 27 patients with CAD, 137 patients without CAD, and 60 patients without screening. Interestingly, the 5-year survival rate was the same for patients with and without CAD (74.9 vs. $74.9 \%$ ). After 5 years, the survival curve of patients with CAD steeply decreased and became close to the same level as the patients without the screening at 10 years (26.7 vs. $27.4 \%$ ) (Fig. 3a). Similar findings were confirmed in the survival curves for cardiac death (Fig. 3b).

\section{Coronary angiography and revascularization}

Coronary angiography was performed within 180 days in 9 (33.3 \%) of 27 with abnormal MPI findings. Seven of nine with coronary angiography had significant CAD that was treated with coronary revascularization therapy (CRT) (one coronary artery bypass surgery and six percutaneous transluminal coronary angioplasty).

\section{Cox proportional hazards model for the factors associated with death}

In the univariate Cox proportional hazards model, age, $\mathrm{SBP}$, serum albumin, $\mathrm{Hb}$, creatinine, eGFR, cCa, CRP, usage of RAS-I, and MPI screening showed significant positive or negative associations with death (Table 3). CRT did not show any significant association with death (Table 3). In the multiple Cox regression analysis, MPI screening was significantly associated with all-cause mortality after adjusting for age, SBP, albumin, hemoglobin, eGFR, calcium, CRP, and usage of RAS-I (Table 4). Furthermore, a significant association with allcause mortality was observed for MPI screening after adjusting the propensity score (Table 4 ).

Age, triglyceride, creatinine, eGFR, corrected Ca, CRP, and MPI screening showed significant positive or negative associations with cardiac death in the univariate Cox proportional hazards model. CRT did not show any significant association with cardiac death. In multiple Cox regression analysis, MPI screening was negatively associated with cardiac death after adjusting for age, eGFR and, CRP (Table 4). Furthermore, a negative association with cardiac death was observed for MPI screening after adjusting the propensity score (Table 4). However, neither of these negative associations was statistically significant.

\section{Discussion}

Main findings of this study

Several studies have observed a high prevalence of CAD at the initiation of dialysis $[8,9]$, with severe CAD having a large influence on poor prognosis after starting dialysis [10]. Therefore, international [11, 12] and Japanese [13] guidelines have recommended that physicians screen patients for coronary heart disease at the

Table 2 Causes of death in the present study

\begin{tabular}{llll}
\hline & Total & MPI screening & \\
\cline { 2 - 4 } & & Yes & No \\
& & $n=164$ & $n=60$ \\
\hline All cause of death & 77 & & 8 \\
Cardiac disease & 17 & 9 & 10 \\
Infectious disease & 22 & 12 & 6 \\
Malignant disease & 14 & 8 & 1 \\
Cerebrovascular disease & 7 & 6 & 4 \\
Other & 14 & 10 & 2 \\
Unknown & 3 & 1 & 4 \\
Cardiac cause death & 17 & 1 & 1 \\
Heart failure & 5 & 3 & 3 \\
Myocardial infarction & 4 & 5 &
\end{tabular}

MPI myocardial perfusion imaging 

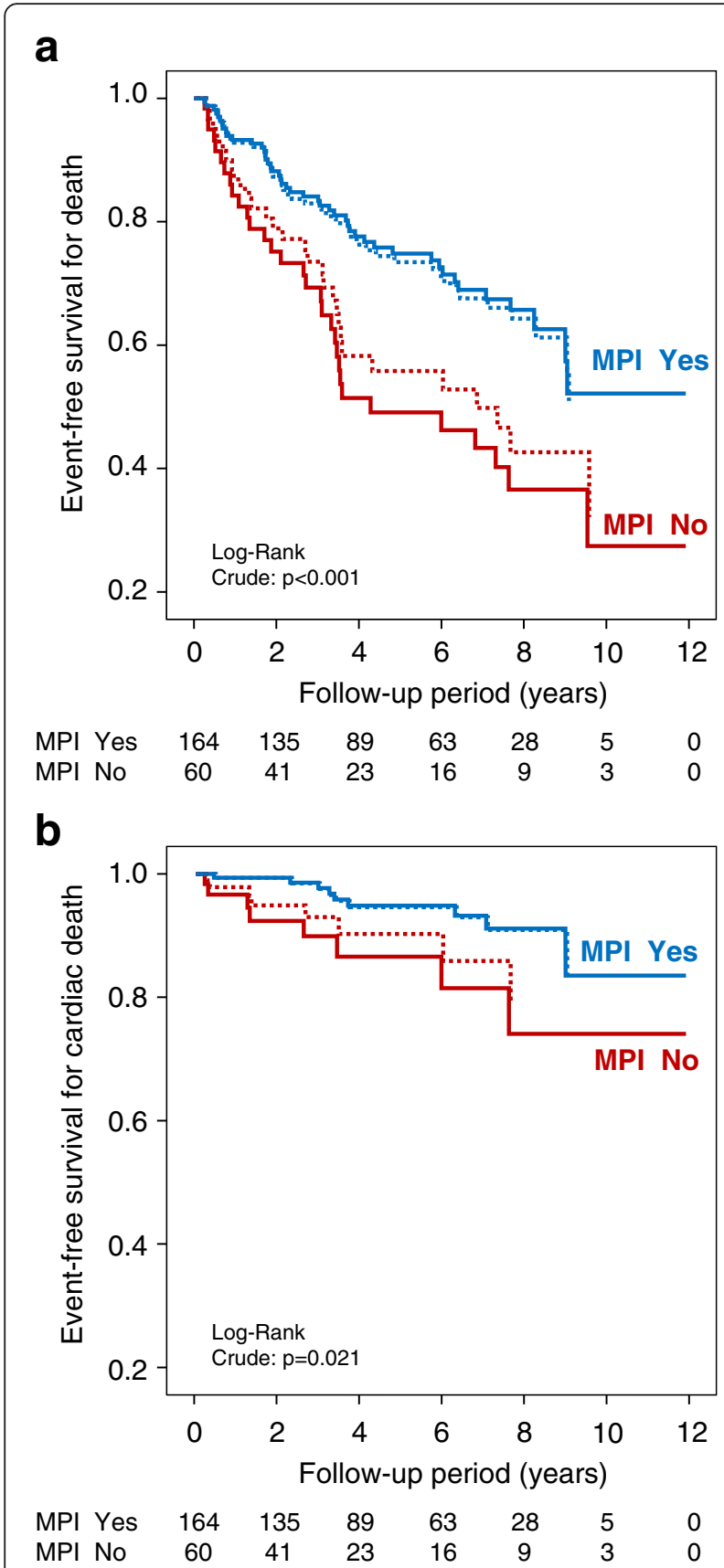

Fig. 2 Comparison of the cumulative survival rate between patients with and without MPI screening. a Patients with MPI screening showed a significantly higher survival rate for all-cause mortality (blue line) compared with patients without the screening (red line) ( $p$ $<0.001$, log-rank test). b A significantly higher survival rate for cardiac death was also observed in patients with MPI screening (blue line) than in patients without screening (red line) ( $p=0.021$, log-rank test). The blue and red dotted lines indicate the propensity scoreadjusted survival curves for each patient group of the same color. Even after adjusting the propensity score, higher survival rates remained for all-cause mortality (a) and cardiac death (b) initiation of dialysis in order to improve their prognosis. However, to date, no evidence has been obtained to prove the effect of screening on prognosis in dialysis patients. The present study demonstrated that MPI screening for $\mathrm{CAD}$ at the initiation of dialysis may lead to improved prognosis in incident dialysis patients without cardiac disease. An approximately 50 \% risk reduction for death produced by the screening was confirmed by using crude cohort analysis, and also by using propensity score adjustment analysis. Our findings may serve as evidence to support the international guidelines $[11,12]$.

\section{Effect of CAD screening on mortality}

Several previous studies have shown the effect of screening on prognosis in the field of cardiovascular disease. Thompson et al. reported the elegant results of their randomized controlled study, in which ultrasound screening for abdominal aortic aneurysm (AAA) resulted in a reduction in all-cause mortality and was beneficial for AAA-related mortality in a population-based sample of men aged 65-74 years [17]. A relative $42 \%$ risk reduction for AAA-related death and $3 \%$ risk reduction in all-cause mortality were confirmed in the screening group compared with the non-screening group. Similarly, Lindholt et al. conducted a randomized singlecenter mass screening trial for AAA [18]. Screening reduced the number of emergency operations by $68 \%$ and lowered the mortality of AAA by $73 \%$ over a 10 -year period in the screening group compared with the control group. Although these reports demonstrate the benefit of screening for AAA in the asymptomatic general population, which is different from the aim of the present study, it is not surprising that the same beneficial findings are confirmed by screening for CAD even in ESKD patients without cardiac disease.

Conversely, several reports have demonstrated that screening for disease had no beneficial effects on mortality. In a randomized controlled trial [19], ultrasound screening for AAA was found to be not effective in men aged 65-83 years and did not reduce overall death rates. Another study [20] showed that echocardiographic screening for structural and valvular heart disease in the general population provided no benefit for mortality or for the risk of myocardial infarction or stroke. The reasons for the contrary outcomes between those studies remain unclear; however, some potential underlying reasons might exist. The prevalence of an implicit target disease for screening in a study cohort would be an important factor affecting the outcome for screening success. If the prevalence of a target cardiovascular disease is very low in a study cohort, there is a high possibility that the screening will not show any beneficial effect if the study cohort is not enough large. Indeed the clinical guideline in American college of physicians has not 


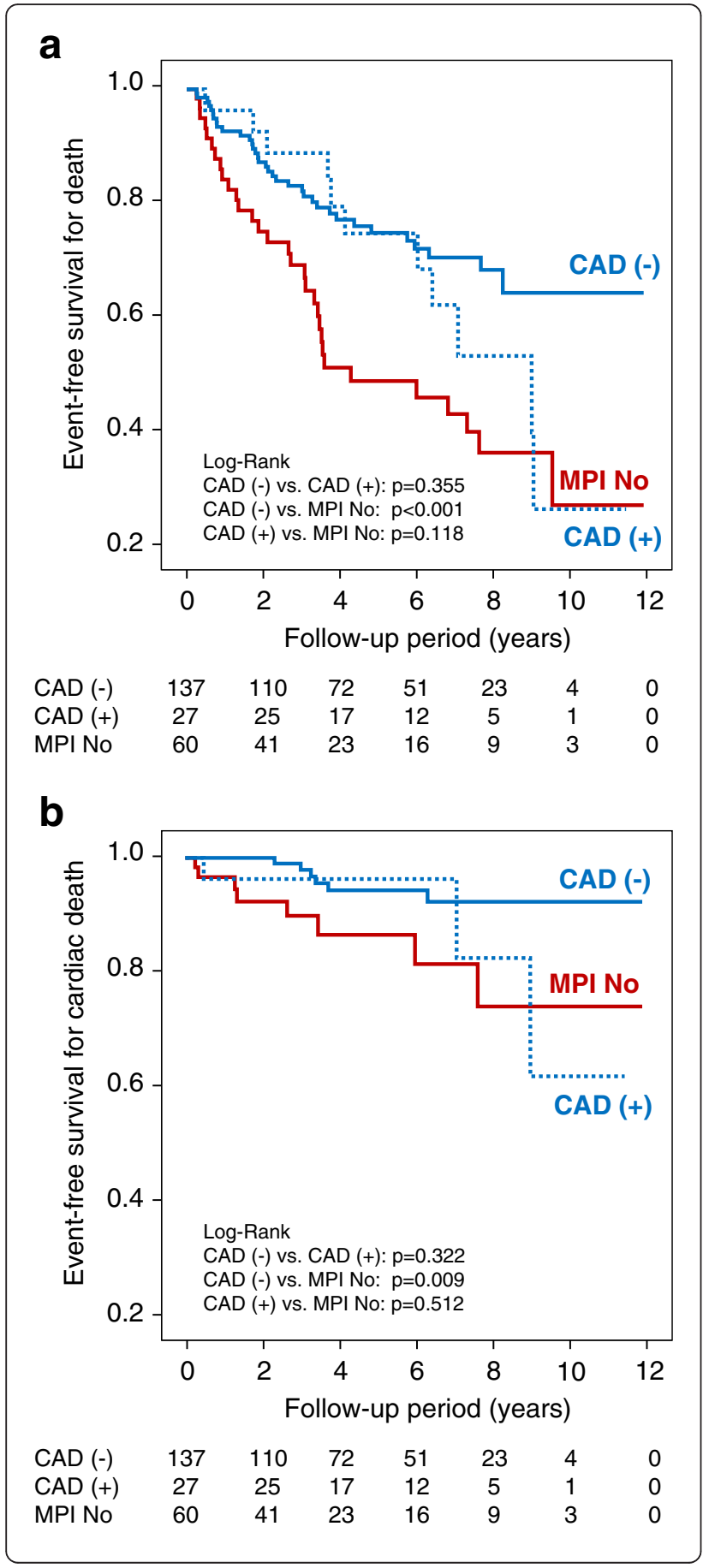

Fig. 3 Comparison of the cumulative survival rate between three patient groups: with $C A D$, without $C A D$, and without MPI screening. a Patients without CAD by MPI screening showed a significantly higher survival rate for all-cause mortality (blue line) compared with patients without screening (red line) ( $p<0.001$, log-rank test). The survival curve for the patients with CAD (dotted blue line) depicts almost the same pattern as the patients without CAD (blue line) until the sixth year of the follow-up period; then, the curve steeply drops to the level of the patients without screening (red line) at 10 years. b A significantly higher survival rate for cardiac death was also observed in patients without CAD by MPI screening (blue line) than in patients without screening (red line) ( $p=0.009$, log-rank test). Similar to the curves for all-cause mortality, the survival curve for the patients with CAD (dotted blue line) shows almost the same pattern as the patients without CAD (blue line) until the sixth year of the follow-up period; then, the curve steeply drops to below the level of the patients without screening (red line) at 10 years

recommended screening for cardiac disease in asymptomatic, low-risk adults with resting or stress electrocardiography, stress echocardiography, or stress myocardial perfusion imaging [21]. The accuracy of a screening test would also play an important role in the study outcome. A lack of sufficient sensitivity and specificity to capture a target disease would crucially influence the results. It is well known that a high prevalence $(40-50 \%)$ of underlying CAD is observed in asymptomatic ESKD patients [8, 9, 22]. Even in ESKD patients, the accuracy of the pharmacological stress MPI for detecting CAD is reported to be sufficiently high, with a sensitivity of $80 \%$ and specificity of $73 \%$ observed in 42 chronic dialysis patients [23] and a sensitivity of $86 \%$ and specificity of $79 \%$ in 80 type I diabetes ESKD patients [24]. Also, the event rate after screening influences the study outcome. Young et al. assessed whether routine screening for CAD by MPI affects cardiac outcomes in asymptomatic type 2 diabetic patients [25]. These investigators concluded that cardiac event rates were not significantly reduced by MPI screening over a follow-up period of 4.8 years due at least in part to a low cardiac event rate of $2.9 \%$. In the present study, during the median followup period of 5.5 years, the cardiac death rate was $22.1 \%$, which is seven times higher than that in the Young study. This is also one of the reasons for the beneficial effect of screening. Therefore, although we used a small sample of ESKD patients in a single-center study, we believe that the findings of the present study provide valuable data.

\section{Greater beneficial effect for all-cause mortality than for cardiac death}

In general, a greater beneficial effect is expected for cardiac death than for all-cause mortality by MPI screening. In the present study, MPI screening had a negative impact on cardiac death; however, this did not reach a statistically significant level in multivariate Cox proportional hazards 
Table 3 Cox proportional hazards model for identifying the factors associated with all-cause mortality and cardiac death

\begin{tabular}{|c|c|c|c|c|c|c|}
\hline & All cau & & & Cardia & ath & \\
\hline & $\mathrm{HR}$ & $95 \% \mathrm{Cl}$ & $p$ value & $\mathrm{HR}$ & $95 \% \mathrm{Cl}$ & $p$ value \\
\hline Age (per year) & 1.053 & $1.032-1.074$ & $<0.001$ & 1.056 & $1.012-1.102$ & 0.012 \\
\hline Male & 1.568 & $0.956-2.573$ & 0.075 & 0.912 & $0.347-2.400$ & 0.852 \\
\hline $\mathrm{BMI}\left(\mathrm{kg} / \mathrm{m}^{2}\right)$ & 0.940 & $0.873-1.014$ & 0.108 & 1.010 & $0.870-1.174$ & 0.893 \\
\hline Diabetes (yes) & 1.119 & $0.715-1.751$ & 0.624 & 0.889 & $0.343-2.309$ & 0.810 \\
\hline $\mathrm{SBP}(\mathrm{mmHg})$ & 0.991 & $0.982-1.000$ & 0.040 & 0.995 & $0.977-1.013$ & 0.559 \\
\hline $\mathrm{DBP}(\mathrm{mmHg})$ & 0.987 & $0.970-1.004$ & 0.121 & 0.988 & $0.954-1.024$ & 0.517 \\
\hline Ejection fraction (\%) & 0.989 & $0.965-1.013$ & 0.355 & 0.957 & $0.914-1.002$ & 0.063 \\
\hline Albumin (g/dL) & 0.503 & $0.307-0.823$ & 0.006 & 0.613 & $0.209-1.792$ & 0.371 \\
\hline Hemoglobin (g/dL) & 1.171 & $1.006-1.363$ & 0.042 & 1.305 & $0.946-1.802$ & 0.105 \\
\hline Total cholesterol (mg/dL) & 1.001 & $0.997-1.006$ & 0.613 & 1.006 & $0.998-1.014$ & 0.156 \\
\hline Triglyceride (mg/dL) & 1.001 & $0.997-1.004$ & 0.701 & 1.004 & $1.000-1.009$ & 0.046 \\
\hline $\mathrm{HDL}-\mathrm{C}(\mathrm{mg} / \mathrm{dL})$ & 0.995 & $0.980-1.010$ & 0.508 & 0.979 & $0.944-1.017$ & 0.275 \\
\hline Creatinin (mg/dL) & 0.818 & $0.745-0.899$ & $<0.001$ & 0.725 & $0.577-0.913$ & 0.006 \\
\hline $\mathrm{eGFR}\left(\mathrm{mL} / \mathrm{min} / 1.73 \mathrm{~m}^{2}\right)$ & 1.292 & $1.166-1.431$ & $<0.001$ & 1.332 & $1.074-1.650$ & 0.009 \\
\hline $\mathrm{cCa}(\mathrm{mg} / \mathrm{dL})$ & 1.303 & $1.027-1.654$ & 0.030 & 1.932 & $1.112-3.359$ & 0.020 \\
\hline$P(\mathrm{mg} / \mathrm{dL})$ & 0.867 & $0.744-1.010$ & 0.067 & 0.783 & $0.560-1.094$ & 0.152 \\
\hline iPTH (pg/mL) & 0.999 & $0.998-1.000$ & 0.155 & 0.999 & $0.996-1.001$ & 0.389 \\
\hline CRP (mg/dL) & 1.29 & $1.055-1.578$ & 0.013 & 1.497 & $1.049-2.138$ & 0.026 \\
\hline Statin (\%) & 1.273 & $0.710-2.282$ & 0.418 & 1.272 & $0.363-4.466$ & 0.707 \\
\hline RAS inhibitor (\%) & 0.522 & $0.333-0.820$ & 0.005 & 0.502 & $0.193-1.307$ & 0.158 \\
\hline Beta blocker (\%) & 1.083 & $0.630-1.861$ & 0.773 & 1.559 & $0.546-4.448$ & 0.407 \\
\hline MPI screening & 0.449 & $0.284-0.708$ & 0.001 & 0.342 & $0.131-0.887$ & 0.027 \\
\hline Coronary artery disease & 1.376 & $0.698-2.716$ & 0.357 & 1.999 & $0.494-8.091$ & 0.332 \\
\hline CRT & 1.102 & $0.274-4.439$ & 0.891 & 2.673 & $0.167-42.794$ & 0.487 \\
\hline
\end{tabular}

$B M I$ body mass index, $C R P$ c-reactive protein, $C R T$ coronary revascularization therapy, SBP systolic blood pressure, DBP diastolic blood pressure, eGFR estimated glomerular filtration rate, $H D L-C$ high-density lipoprotein cholesterol, RAS renin angiotensin system

analysis. Few cardiac death events during the follow-up period may lead to insufficient statistical power for analysis. However, a possible explanation can be raised for this issue. The first possible explanation is that the patients with MPI screening may be a special sub-cohort who pays more attention to their own health, compared to the patients without the screening. Although it is a matter of speculation, screening for CAD at the initiation of dialysis could be a trigger to alert patients to be vigilant regarding their overall health. This would be a reason why the screening might exert a more beneficial effect on improving prognosis for all-cause mortality than for cardiac death.

The second possible explanation may relate to the results of our previous study [26]. In that study, we analyzed the survival rates for major adverse cardiac events in incident dialysis patients without CAD and found that even in patients without significant CAD, cardiac events occurred after 1 year of starting dialysis. We have proposed to administer a second screening for CAD at least 2 years after starting dialysis, even if no significant $C A D$ was confirmed at the initiation of dialysis. It is

Table 4 Freedom from events of MPI

\begin{tabular}{|c|c|c|c|c|c|c|}
\hline & \multicolumn{3}{|l|}{ Crude } & \multicolumn{3}{|c|}{ Propensity score adjusted } \\
\hline & $\mathrm{HR}$ & $95 \% \mathrm{Cl}$ & $p$ value & $\mathrm{HR}$ & $95 \% \mathrm{Cl}$ & $p$ value \\
\hline All cause of death & $0.581^{a}$ & $0.352-0.959^{a}$ & $0.034^{a}$ & 0.568 & $0.347-0.932$ & 0.025 \\
\hline Cardiac death & $0.419^{b}$ & $0.157-1.118^{b}$ & $0.082^{b}$ & 0.491 & $0.175-1.377$ & 0.177 \\
\hline
\end{tabular}

CRP c-reactive protein, $S B P$ systolic blood pressure, eGFR estimated glomerular filtration rate, $R A S$ renin angiotensin system

${ }^{a}$ Adjusted by age, SBP, albumin, hemoglobin, eGFR, calcium, CRP, and RAS inhibitor

${ }^{\mathrm{b}}$ Adjusted by age, eGFR, and CRP, MPI myocardial perfusion imaging 
conceivable that a higher number of physician visits may be made by patients who underwent a first screening compared with the patients without screening; consequently, the mortality rate may decrease in the patients who undergo screening for CAD.

The third possible explanation is attempts to lower the rate of cardiac death. Twenty-seven patients among the $164(16.4 \%)$ who received screening suffered from CAD in the present study. Physicians may pay more attention and add intensive treatment to those patients to improve prognosis. In fact, it was found that the 5-year survival rate of the 27 ESKD patients with CAD was $74.9 \%$, which is not inferior to the 5-year survival rate of $74.9 \%$ in the 137 ESKD patients without CAD. The 1-year survival rate showed a large difference (26.7 vs. $64.5 \%$ ) between patients with and without CAD. On the other hand, in the 60 ESKD patients without the screening, approximately 10 patients (about $16.5 \%$ ) were calculated to have suffered from CAD if the same prevalence in the 164 ESKD patients with the screening had occurred. Failure to pay attention and insufficient management in those 10 patients may have played a role, at least in part, in poor prognosis for all-cause mortality. However, the possible advantages of screening on prognosis could not be fully explained based only on the difference in management of CAD.

The fourth possible explanation of greater benefit for all-cause mortality than for cardiac death is the potential for selection bias. The design of our study was an observational cohort study, not a randomized prospective study. It cannot be denied that relatively low-risk patients were selected and underwent the screening by chance. In order to minimize the selection bias, according to the analytical technique used in the present study, we calculated the propensity score by using all background factors to adjust for differences in baseline characteristics. As shown in Table 4, an approximately $50 \%$ risk reduction for death engendered by the screening appeared even after adjusting the propensity score. Therefore, it is more plausible that screening at the initiation of dialysis could lead to a better prognosis for incident dialysis than ever before.

\section{The timing of screening in CKD patients}

To improve the prognosis of hemodialysis patients, the best goal we should reach is to start dialysis without any cardiovascular complication. Therefore, we think that the screening should be performed in earlier predialytic phase of CKD such as CKD 3 or 4. The initiation of HD as timing for screening may be too late. This would be a reason why the beneficial effect of MPI screening at the initiation of dialysis on cardiac mortality in this study showed marginal.

\section{Limitations}

The present study had some limitations. In order to accurately evaluate the effects of screening on prognosis, a prospective randomized controlled study is the gold standard. However, based on the recommendations of international guidelines [11, 12], it is impossible to plan a study in which the patients are randomly divided according to whether screening is performing. We felt constrained to use the propensity score adjustment method to prove our hypothesis. Another limitation was that this was a single-center observational study with a relatively small number of patients. A larger-scale study should be conducted in the future.

\section{Conclusions}

Screening for CAD at the initiation of dialysis could lead to better prognosis after starting dialysis. This finding is considered to be in line with the recommendation of international guidelines [11, 12]. A larger-scale randomized controlled study is needed to confirm this finding.

\section{Competing interests}

$\mathrm{NJ}$ received lecture fee from CHUGAI Pharmaceutical and KYOWA KIRIN and received research funding from Roche Diagnostics. The other authors declare that they have no competing interests.

\section{Authors' contributions}

$\mathrm{HH}$ and NJ had full access to all the data in the study and take responsibility for the integrity of the data and analytical accuracy. $\mathrm{HH}, \mathrm{NJ}$, and YT contributed to the study concept and design. MI, SK, TA, YT, YI, AM, MH, HN, and $\mathrm{KH}$ participated in the data acquisition. $\mathrm{YT}, \mathrm{TH}, \mathrm{HN}$, and $\mathrm{NJ}$ performed the data analysis and interpretation. $\mathrm{YT}$ and $\mathrm{NJ}$ drafted the manuscript. $\mathrm{YT}$, $\mathrm{TH}$, and NJ were involved in the statistical analysis. KH gave administrative, technical, and material support. All authors read and approved the final manuscript.

\section{Acknowledgements}

The authors greatly appreciate the staff of the Cardiac Nuclear Medicine Department at Toho University Ohashi Medical Center for their evaluations of the myocardial perfusion imaging studies.

\section{Author details}

'Department of Nephrology, Toho University Ohashi Medical Center, 2-17-6 Ohashi, Meguro-ku, Tokyo 153-8515, Japan. ²Dialysis Center, Nissan Tamagawa Hospital, Tokyo, Japan. ${ }^{3}$ Hirahata Clinic, Tokyo, Japan.

${ }^{4}$ Department of Cardiovascular Medicine, Toho University Ohashi Medical Center, Tokyo, Japan.

Received: 13 December 2015 Accepted: 16 March 2016 Published online: 01 June 2016

References

1. Ronco C, McCullough P, Anker SD, Anand I, Aspromonte N, Bagshaw SM, et al. Cardio-renal syndromes: report from the consensus conference of the acute dialysis quality initiative. Eur Heart J. 2010;31:703-11.

2. Investigators ET, Chertow GM, Block GA, Correa-Rotter R, Drueke TB, Floege $J$, et al. Effect of cinacalcet on cardiovascular disease in patients undergoing dialysis. N Engl J Med. 2012;367:2482-94.

3. Wheeler DC, London GM, Parfrey PS, Block GA, Correa-Rotter R, Dehmel B, et al. Effects of cinacalcet on atherosclerotic and nonatherosclerotic cardiovascular events in patients receiving hemodialysis: the EValuation Of Cinacalcet $\mathrm{HCl}$ Therapy to Lower CardioVascular Events (EVOLVE) trial. J Am Heart Assoc. 2014;3:e001363.

4. Moroi M, Tamaki N, Nishimura M, Haze K, Nishimura T, Kusano E, et al, Association between abnormal myocardial fatty acid metabolism and 
cardiac-derived death among patients undergoing hemodialysis: results from a cohort study in Japan. Am J Kidney Dis. 2013;61:466-75.

5. Shoji T, Emoto M, Tabata T, Kimoto E, Shinohara K, Maekawa K, et al. Advanced atherosclerosis in predialysis patients with chronic renal failure. Kidney Int. 2002;61:2187-92.

6. Hase H, Tsunoda T, Tanaka Y, Takahashi Y, Imamura Y, Ishikawa H, et al. Risk factors for de novo acute cardiac events in patients initiating hemodialysis with no previous cardiac symptom. Kidney Int. 2006;70:1142-8.

7. Tanaka $\mathrm{Y}$, Joki N, Hase H. History of acute coronary events during the predialysis phase of chronic kidney disease is a strong risk factor for major adverse cardiac events in patients initiating haemodialysis. Nephrol Dial Transplant. 2007;22:2917-23.

8. Joki N, Hase H, Nakamura R, Yamaguchi T. Onset of coronary artery disease prior to initiation of haemodialysis in patients with end-stage renal disease. Nephrol Dial Transplant. 1997:12:718-23.

9. Ohtake T, Kobayashi S, Moriya H, Negishi K, Okamoto K, Maesato K, et al. High prevalence of occult coronary artery stenosis in patients with chronic kidney disease at the initiation of renal replacement therapy: an angiographic examination. J Am Soc Nephrol. 2005;16:1141-8.

10. Joki N, Hase H, Takahashi Y, Ishikawa H, Nakamura R, Imamura Y, et al. Angiographical severity of coronary atherosclerosis predicts death in the first year of hemodialysis. Int Urol Nephrol. 2003;35:289-97.

11. K/DOQI. Clinical practice guidelines for cardiovascular disease in dialysis patients. Am J Kidney Dis. 2005;45:S1-S153.

12. Herzog CA, Asinger RW, Berger AK, Charytan DM, Diez J, Hart RG, et al. Cardiovascular disease in chronic kidney disease. A clinical update from Kidney Disease: Improving Global Outcomes (KDIGO). Kidney Int. 2011;80: 572-86.

13. Hirakata H, Nitta K, Inaba M, Shoji T, Fujii H, Kobayashi S, et al. Japanese Society for Dialysis Therapy guidelines for management of cardiovascular diseases in patients on chronic hemodialysis. Ther Apher Dial. 2012;16:387435.

14. Hase H, Joki N, Ishikawa H, Fukuda H, Imamura Y, Saijyo T, et al. Prognostic value of stress myocardial perfusion imaging using adenosine triphosphate at the beginning of haemodialysis treatment in patients with end-stage renal disease. Nephrol Dial Transplant. 2004;19:1161-7.

15. Joki N, Hase H, Kawano Y, Nakamura S, Nakajima K, Hatta T, et al. Myocardial perfusion imaging for predicting cardiac events in Japanese patients with advanced chronic kidney disease: 1-year interim report of the J-ACCESS 3 investigation. Eur J Nucl Med Mol Imaging. 2014:41:1701-9.

16. Matsuo S, Imai E, Horio M, Yasuda Y, Tomita K, Nitta K, et al. Revised equations for estimated GFR from serum creatinine in Japan. Am J Kidney Dis. 2009;53:982-92.

17. Thompson SG, Ashton HA, Gao L, Buxton MJ, Scott RA, Multicentre Aneurysm Screening Study G. Final follow-up of the Multicentre Aneurysm Screening Study (MASS) randomized trial of abdominal aortic aneurysm screening. Br J Surg. 2012;99:1649-56.

18. Lindholt JS, Juul S, Fasting H, Henneberg EW. Preliminary ten year results from a randomised single centre mass screening trial for abdominal aortic aneurysm. Eur J Vasc Endovasc Surg. 2006;32:608-14.

19. Norman PE, Jamrozik K, Lawrence-Brown MM, Le MT, Spencer CA, Tuohy RJ, et al. Population based randomised controlled trial on impact of screening on mortality from abdominal aortic aneurysm. BMJ. 2004;329:1259.

20. Lindekleiv H, Lochen ML, Mathiesen EB, Njolstad I, Wilsgaard T, Schirmer H. Echocardiographic screening of the general population and long-term survival: a randomized clinical study. JAMA Intern Med. 2013;173:1592-8.

21. Chou R, High Value Care Task Force of the American College of P. Cardiac screening with electrocardiography, stress echocardiography, or myocardial perfusion imaging: advice for high-value care from the american college of physicians. Ann Intern Med. 2015;162:438-47.

22. Charytan $D$, Kuntz RE, Mauri L, DeFilippi C. Distribution of coronary artery disease and relation to mortality in asymptomatic hemodialysis patients. Am J Kidney Dis. 2007;49:409-16.

23. Dahan M, Legallicier B, Himbert D, Faraggi M, Aubry N, Siohan P, et al. Diagnostic value of myocardial thallium stress scintigraphy in the detection of coronary artery disease in patients undergoing chronic hemodialysis. Arch Mal Coeur Vaiss. 1995;88:1121-3.

24. Boudreau RJ, Strony JT, duCret RP, Kuni CC, Wang Y, Wilson RF, et al. Perfusion thallium imaging of type I diabetes patients with end stage renal disease: comparison of oral and intravenous dipyridamole administration. Radiology. 1990;175:103-5.
25. Young LH, Wackers FJ, Chyun DA, Davey JA, Barrett EJ, Taillefer R, et al. Cardiac outcomes after screening for asymptomatic coronary artery disease in patients with type 2 diabetes: the DIAD study: a randomized controlled trial. JAMA. 2009:301:1547-55.

26. Joki N, Tanaka Y, Ishikawa H, Takahashi Y, Iwakura Y, Masuda H, et al. Optimum second screening point for detection of coronary artery disease in hemodialysis patients without advanced coronary artery disease. Am J Nephrol. 2009;29:420-5.

\section{Submit your next manuscript to BioMed Central and we will help you at every step:}

- We accept pre-submission inquiries

- Our selector tool helps you to find the most relevant journal

- We provide round the clock customer support

- Convenient online submission

- Thorough peer review

- Inclusion in PubMed and all major indexing services

- Maximum visibility for your research

Submit your manuscript at www.biomedcentral.com/submit
C Biomed Central 